\title{
Effects of prenatal $n-3$ fatty acid supplementation on offspring resolvins at birth and 12 years of age: a double-blind, randomised controlled clinical trial
}

\author{
Valene H. L. See ${ }^{1,2}$, Emilie Mas ${ }^{1}$, Susan L. Prescott ${ }^{2,3}$, Lawrence J. Beilin ${ }^{1}$, Sally Burrows ${ }^{1}$, \\ Anne E. Barden ${ }^{1}$, Rae-Chi Huang ${ }^{3} \dagger$ and Trevor A. Mori ${ }^{1 * \dagger}$ \\ ${ }^{1}$ School of Medicine, Royal Perth Hospital, The University of Western Australia, Perth, WA 6000, Australia \\ ${ }^{2}$ School of Paediatrics and Child Health, The University of Western Australia, Perth, WA 6008, Australia \\ ${ }^{3}$ Telethon Kid's Institute, The University of Western Australia, Perth, WA 6008, Australia
}

(Submitted 12 September 2016 - Final revision received 29 September 2017 - Accepted 5 October 2017)

\section{Abstract}

Resolution of inflammation is an active process involving specialised pro-resolving mediators (SPM) generated from the $n$ - 3 fatty acids EPA and DHA. $n$-3 Fatty acid supplementation during pregnancy may provide an intervention strategy to modify these novel SPM. This study aimed to assess the effect of $n-3$ fatty acid supplementation in pregnancy on offspring SPM at birth and 12 years of age (12 years). In all, ninety-eight atopic pregnant women were randomised to $3.7 \mathrm{~g}$ daily $n-3$ fatty acids or a control (olive oil), from 20 weeks gestation until delivery. Blood was collected from the offspring at birth and at 12 years. Plasma SPM consisting of 18-hydroxyeicosapentaenoic acid (18-HEPE), E-series resolvins, 17-hydroxydocosahexaenoic acid (17-HDHA), D-series resolvins, 14-hydroxydocosahexaenoic acid (14-HDHA), 10 S,17S-dihydroxydocosahexaenoic acid, maresins and protectin 1, were measured by liquid chromatography-tandem MS. We identified the resolvins RvE1, RvE2, RvE3, RvD1, 17R-RvD1 and RvD2 for the first time in human cord blood. $n$ - 3 Fatty acids increased cord blood 18-HEPE $(P<0.001)$ derived from EPA relative to the control group. DHA-derived 17-HDHA at birth was significantly increased in the $n$-3 fatty acid group relative to the controls $(P=0 \cdot 001)$, but other SPM were not different between the groups. $n-3$ Fatty acid supplementation during pregnancy was associated with an increase in SPM precursors in the offspring at birth but the effects were not sustained at 12 years. The presence of these SPM, particularly at birth, may have functions relevant in the newborn that remain to be established, which may be useful for future investigations.

Key words: $\boldsymbol{n}$-3 Fatty acid supplementation: Pregnancy: Resolvins: Protectins: Inflammation

Inflammation plays an important role in acute infection and injury, but it can be harmful in chronic non-communicable diseases. Mitigation of the harmful effects of oxidative stress and resolution of inflammation is an active process regulated by biochemical mediators and receptor-signalling pathways, driven by specialised pro-resolving mediators (SPM) ${ }^{(1)}$. These enzymatically produced SPM exert potent receptor-mediated immuno-resolving effects on the activated immune system to resolve inflammation by restoring homoeostasis ${ }^{(2)}$. Studies have shown that SPM increase with time during the inflammatory process, acting on a number of $\mathrm{G}$-coupled protein receptors, including the leukotriene $\mathrm{B}_{4}\left(\mathrm{LTB}_{4}\right)$ receptor to affect resolution of inflammation ${ }^{(1,3)}$.

Serhan \& Petasis ${ }^{(1)}$ first described SPM derived from the $n-3$ fatty acids EPA and DHA. SPM derived from EPA via the intermediate 18-hydroxyeicosapentaenoic acid (18-HEPE) are known as E-series resolvins, whereas those derived from DHA via 17-hydroxydocosahexaenoic acid (17-HDHA) are known as D-series resolvins ${ }^{(1)}$. Protectins (PD1) and maresins (MaR-1) are derived from DHA via the precursor 17-hydroperoxydocosahexaenoic acid (17-HpDHA) and 14-hydroxydocosahexaenoic acid (14-HDHA), respectively ${ }^{(1)}$.

SPM are present in biological fluids, cells and tissues and increase with time during the inflammatory process ${ }^{(1,4)}$. To date most of the characterisation of SPM has been reported in animal models of inflammation ${ }^{(4)}$. A number of SPM have been reported in human blood at concentrations previously shown to be biologically active (i.e. $0 \cdot 1-0 \cdot 5 \mathrm{~nm})^{(5)}$ but limited data are available on SPM in human tissues and their role and significance in inflammatory pathophysiology. In animal models, SPM have been shown to have antiviral properties ${ }^{(6)}$, reduce $\operatorname{arthritic}^{(7)}$ and post-operative pain $^{(8)}$, colitis $^{(9)}$, airway

Abbreviations: 14-HDHA, 14-hydroxydocosahexaenoic acid; 17-HDHA, 17-hydroxydocosahexaenoic acid; 18-HEPE, 18-hydroxyeicosapentaenoic acid; MLE, maximum likelihood estimation; OO, olive oil; PD1, protectin 1; Rv, resolvin; SPM, specialised pro-resolving mediators.

* Corresponding author: Trevor A. Mori, fax +6189224 0246, email trevor.mori@uwa.edu.au

$\dagger$ Equal senior authorship. 
inflammation $^{(10,11)}$, ocular inflammation ${ }^{(12)}$ and pneumonia ${ }^{(13)}$, and promote tissue regeneration ${ }^{(14)}$. Studies in rodents show that maternal dietary $n-3$ fatty acid intake increased SPM and the upstream precursors in the placenta ${ }^{(15)}$. However, maternal $n-3$ fatty acid supplementation in human studies only increased the precursors 18-HEPE and 17-HDHA in the placenta ${ }^{(16)}$.

The 'developmental origins of health and disease' (DOHaD) hypothesis proposes that factors that modify physiological processes during the critical developmental period in early life may exert a long-term influence on disease in adult life ${ }^{(17)}$. Thus, early life nutritional exposures may be significant determinants of the development and future health of the offspring. In this regard, maternal $n-3$ fatty acid supplementation in pregnancy has been shown to enhance $n-3$ fatty acid status of the offspring ${ }^{(18)}$, influence immunological outcomes by modifying offspring cytokine concentrations ${ }^{(19,20)}$ and progenitor phenotype ${ }^{(21)}$, attenuate offspring lipid peroxidation ${ }^{(22)}$ and improve early childhood cognitive and visual development ${ }^{(23)}$.

To our knowledge, there are no data on SPM concentrations in offspring at birth and early childhood following maternal $n$-3 fatty acid supplementation during pregnancy. Therefore, this study aimed to examine the effects of maternal $n$ - 3 fatty acid supplementation during pregnancy on plasma SPM and their precursors in the offspring. We hypothesised that maternal n-3 fatty acid supplementation would increase cord SPM with consequent benefit to the fetus and the offspring at birth, and that there would be a programming effect such that these benefits would be sustained to 12 years of age (12 years)

\section{Methods}

\section{Recruitment of participants}

Details relating to the study design, methodology, inclusion and exclusion criteria have been published ${ }^{(19)}$. In brief, the study was a single-centred, randomised (1:1), double-blind, placebo-controlled parallel study with participants stratified by parity (no previous term childbirth $v$. one or more), prepregnancy BMI, age and maternal allergy (allergic rhinitis or asthma). The original study aimed to investigate the effects of maternal $n$ - 3 fatty acid supplementation during pregnancy on neonatal immune development, allergy and neurodevelopment outcomes in the offspring $^{(18-20,22-25)}$. A follow-up was undertaken at 12 years to assess the effects of $n-3$ fatty acid supplementation in pregnancy on SPM in childhood. The study was registered with the Australian Clinical Trials Registry (ACTRN00336395, https://www. anzctr.org.au/Trial/Registration/TrialReview.aspx?id=336395).

In all, ninety-eight women with a history of allergy, and booked for delivery at St. John of God Hospital, Subiaco, Western Australia were recruited and randomised between January 1999 and September 2001. The women were otherwise healthy, non-smokers and had their fish intake restricted. Maternal allergy was defined by a positive skin prick test and a history of physician-diagnosed allergic rhinitis and/or asthma. The study was carried out in accordance with the guidelines laid down in the Declaration of Helsinki and all procedures were approved by the St John of God Hospital and Princess
Margaret Hospital Human Ethics Committees. Written informed consent was obtained from all mothers.

\section{Intervention}

The women were randomised to receive either $4 \times 1 \mathrm{~g}$ fish oil or olive oil (OO) capsules as the control. Supplementation commenced at 20 weeks of gestation and ceased at delivery. The $n-3$ fatty acid capsules (Ocean Nutrition Ltd) provided a total of $3.7 \mathrm{~g}$ of $n$-3 fatty acids as ethyl esters with $56.0 \%$ as DHA and $27.7 \%$ as EPA (confirmed by GC). Placebo capsules (Pan Laboratories) contained OO with $66.6 \%$ as $n$-9 oleic acid and $<1 \% n$-3 PUFA. This dose was based on previous trials studying the effect of $n-3$ fatty acid supplementation in pregnancy ${ }^{(26)}$, on high-risk pregnancies ${ }^{(27)}$, on blood pressure ${ }^{(28)}$ and in our previous studies in adults $^{(29)}$. Randomisation and dispensing of capsules were conducted at a different centre and independent of those involved with co-ordinating the trial. Participants, research scientists, and the paediatrician remained blinded to the group allocation for the duration of the study. The participants completed a validated, semi-quantitative FFQ at 20- and 30-weeks gestation to monitor fish consumption. Compliance with the treatment regimen was checked by measuring the incorporation of DHA and EPA into erythrocyte cell membranes of the women at 30- and 36-weeks gestation and 6 weeks postnatally ${ }^{(18)}$.

\section{Outcomes}

The offspring were assessed at birth and 12 years for resolvins, protectins and maresins and their precursors. At birth, 83 infants were included in the study: eighty-one cord blood samples were available for analyses. At 12 years, fifty-eight children participated in the follow-up study: forty-three blood samples were available for analyses.

\section{Clinical outcomes}

Infants were clinically evaluated at 12 months of age (1 year), which included a detailed history and clinical examination ${ }^{(19)}$. The clinical outcome measures considered in this study were incidence of atopic eczema dermatitis syndrome, food allergy and allergic disease.

\section{Blood collection and processing}

Cord blood was collected at birth from the placental vessels. Peripheral blood was obtained by venepuncture after an overnight fast at 12 years, where consented and practical. Blood was collected into heparinised tubes and processed immediately after the clinic visit ${ }^{(19)}$. Plasma was stored at $-80^{\circ} \mathrm{C}$. Erythrocytes were washed before lipids were extracted with methanol-chloroform (2:1) and stored at $-80^{\circ} \mathrm{C}$.

\section{Fatty acid measurements}

Fatty acid ethyl esters were extracted from washed red cell membranes and assayed by GC as previously described for cord erythrocytes $^{(30)}$ and erythrocytes at 12 years ${ }^{(31)}$. 
Measurement of specialised pro-resolving mediators in plasma

SPM standards were purchased from Cayman Chemicals ${ }^{(32,33)}$. 10R,17S-dihydroxy-4Z,7Z,11E,13E, 15Z,19Z-DHA (PD1) standard was a gift by Professor Charles N. Serhan (Harvard Medical School). 5S,18R-dihydroxy-6E,8Z,11Z,14Z,16E-EPA (resolvin E2 (RvE2)), (17,18S-dihydroxy-5Z,8Z,11Z,13E,15E- EPA (RvE3) and 17,18R-dihydroxy-5Z,8Z,11Z,13E,15E-EPA (18R-RvE3) standards were a gift from Professor Makoto Arita (Department of Health Chemistry, Graduate School of Pharmaceutical Sciences, University of Tokyo). 5S,12R,18R-trihydroxy-6Z,8E,10E,14Z,16EEPA (RvE1) and (14-hydroxy-4Z,7Z,10Z,12E,16Z,19Z-DHA (14-HDHA) were provided by Cayman Chemicals.

The assay was performed as previously described ${ }^{(32,33)}$. In brief, $1 \mathrm{ml}$ of plasma and internal standard $\mathrm{LTB}_{4}-\mathrm{d}_{4}(500 \mathrm{pg})$ were acidified with $2 \mathrm{ml}$ of $100 \mathrm{~mm}$ sodium acetate $\mathrm{pH} 3$, applied to solid phase extraction cartridges (Bond Elut C18 $500 \mathrm{mg}$; Agilent Technologies), and washed with water and hexane. The SPM were eluted with ethyl acetate $(2 \mathrm{ml})$, dried under $\mathrm{N}$ and reconstituted in $100 \mathrm{ml}$ of $5 \mathrm{~mm}$ ammonium acetate ( $\mathrm{pH}$ 8.9) and methanol (50/50; v/v) for analysis by liquid chromatography-tandem MS. The SPM were quantitated using a Thermo Scientific TSQ Quantum Ultra Triple Quadrupole LC-MS System (ThermoFisher Scientific) equipped with an electrospray ionisation source operated in negative ion mode. The detection limit for 18-HEPE, 17-HDHA, and 14-HDHA was $10 \mathrm{pg} / \mathrm{ml}$ and the detection limit for other SPM was $20 \mathrm{pg} / \mathrm{ml}$. We extracted a larger volume of plasma for SPM analysis for samples that gave results close to the limit of detection. Instrument control and data acquisition were performed using the Xcalibur 2.0.7 software.

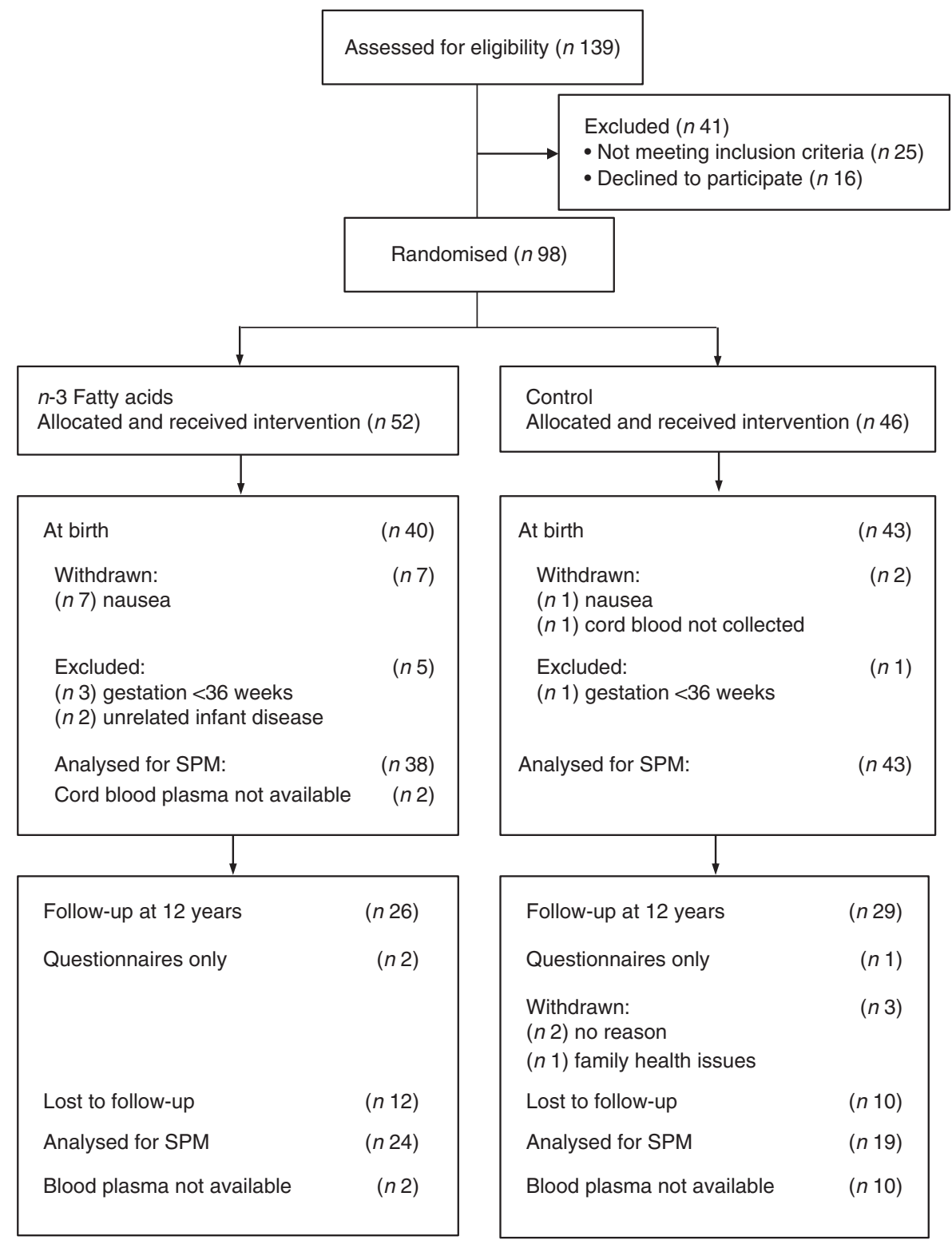

Fig. 1. The CONSORT diagram showing volunteer recruitment and randomisation. 


\section{Statistical analysis}

Data were summarised using counts, percentages, means and standard deviations as appropriate. Log transformations were applied to outcomes when the data were not approximately normal. For these variables, geometric means and standard deviations were calculated. Comparisons of characteristics of children with and without blood samples were performed using chi-square tests for dichotomous data. For continuous data, group differences were assessed by $t$ tests for normal data and Mann-Whitney $U$ tests for data that could not achieve normality.

Due to insufficient data for RvE2 and RvE3 at 12 years, these variables were not utilised for analysis. Group differences at birth for RvE2 and RvE3 were analysed using Tobit regression. Tobit regression considers the probability of the data being below (or above) the detection limit and uses this in conjunction with data available above the limit; hence, those with values below the limit are not excluded. The effect of $n$ - 3 fatty acids on SPM concentrations between birth and 12 years was

Table 1. Characteristics of the study population

(Mean values and standard deviations; numbers and percentages) examined using linear mixed models with maximum likelihood estimation (MLE) to produce unbiased estimates (mixed-effects maximum likelihood regression). MLE handles missing data by using observed data as well as intra-class correlation to inform the likelihood function which is then maximised to obtain an estimate of the mean at a given time point. MLE therefore retains participants if data is provided for at least one time point, thus avoiding attrition bias. Outcomes with a detection limit at birth and at 12 years (RvE1 and RvD2) were assessed using random effects tobit analysis. Where normality of the data could not be met for repeated measures (RvD1 and 17R-RvD1), quantile regression with a per-person cluster adjustment was performed. Differences in the pattern over time between intervention groups were tested by the interaction of group and time in the model. In each model, interaction of group and time was used to test for a difference between groups in the change over time. The Bonferroni-Holm stepdown process was employed to adjust $P$ values for multiple testing.

Associations between SPM and $n$ - 3 fatty acids were investigated with three-way and two way interactions of group, year

\begin{tabular}{|c|c|c|c|c|}
\hline \multirow[b]{2}{*}{ Characteristics } & \multicolumn{2}{|c|}{$\begin{array}{l}n-3 \text { Long-chain PUFA group } \\
(n 38)\end{array}$} & \multicolumn{2}{|c|}{$\begin{array}{l}\text { Olive oil group } \\
\quad(n 43)\end{array}$} \\
\hline & Mean $/ n$ & $\mathrm{sD} / \%$ & Mean/n & $\mathrm{SD} / \%$ \\
\hline \multicolumn{5}{|l|}{ Antenatal } \\
\hline Gestational age (d) & 274.9 & 7.5 & 274.2 & 7.8 \\
\hline Prepregnancy BMI (kg/m²) & 23.4 & 3.4 & $24 \cdot 1$ & 4.0 \\
\hline Maternal age (years) & $31 \cdot 1$ & $4 \cdot 0$ & $32 \cdot 4$ & 3.5 \\
\hline \multicolumn{5}{|l|}{ Maternal allergic status } \\
\hline Asthma & 17 & $44 \cdot 7$ & 17 & 39.5 \\
\hline Rhinitis & 21 & $55 \cdot 3$ & 26 & 60.5 \\
\hline \multicolumn{5}{|l|}{ Parity } \\
\hline \multirow{2}{*}{\multicolumn{5}{|c|}{ Delivery method }} \\
\hline & & & & \\
\hline Normal labour & 27 & $71 \cdot 1$ & 35 & $81 \cdot 4$ \\
\hline \multicolumn{5}{|l|}{ Maternal smoked before } \\
\hline Yes & 13 & $34 \cdot 2$ & 21 & $48 \cdot 8$ \\
\hline \multicolumn{5}{|l|}{ Maternal passive smoking } \\
\hline Yes & 21 & $55 \cdot 3$ & 24 & $55 \cdot 8$ \\
\hline \multicolumn{5}{|l|}{ Partner smoking status } \\
\hline Yes & 5 & 13.2 & 4 & $9 \cdot 3$ \\
\hline \multicolumn{5}{|l|}{ Maternal education (years) } \\
\hline $10-12$ & 15 & 39.5 & 9 & $20 \cdot 9$ \\
\hline$>12$ & 23 & $60 \cdot 5$ & 34 & $79 \cdot 1$ \\
\hline \multicolumn{5}{|l|}{ Birth } \\
\hline \multicolumn{5}{|l|}{ Sex } \\
\hline Male & 16 & $42 \cdot 1$ & 22 & $51 \cdot 2$ \\
\hline Birth weight (g) & 3522 & 279 & 3430 & 372 \\
\hline Birth length $(\mathrm{cm})$ & 50.4 & $2 \cdot 0$ & $49 \cdot 7$ & 1.9 \\
\hline Head circumference $(\mathrm{cm})$ & $35 \cdot 2^{*}$ & 1.3 & 34.7 & 1.2 \\
\hline Apgar score & $8 \dagger$ & 1 & $8 \ddagger$ & 1 \\
\hline \multicolumn{5}{|l|}{ Infant } \\
\hline \multicolumn{5}{|l|}{ Breast-fed at 6 weeks } \\
\hline Yes & 32 & $84 \cdot 2$ & 36 & $87 \cdot 8$ \\
\hline \multicolumn{5}{|l|}{ Breast-fed at 6 months } \\
\hline Yes & 24 & 64.9 & 29 & $67 \cdot 4$ \\
\hline \multicolumn{5}{|l|}{ Breast-fed at 1 year } \\
\hline Yes & 11 & 29.0 & 11 & $25 \cdot 6$ \\
\hline Duration of breast-feeding (d) & 239 & 139 & 219 & 138 \\
\hline
\end{tabular}

* Data available for $n 37$

† Data available for $n 29$

‡ Data available for $n 34$ 
and the variable of interest. Erythrocyte fatty acids were analysed using linear mixed models with MLE.

A $P$ value of $<0.05$ (two-tailed) was considered statistically significant for all analyses. All statistical analyses were performed using the Stata 13.1 statistical package (StataCorp).

\section{Statistical power}

The number of subjects was based on previous studies by the host laboratory comparing cord blood immune responses of neonates with or without later allergy ${ }^{(19)}$. It was estimated that the sample size of eighty-three subjects would give $>90 \%$ power to detect a $58 \%$ difference in the SPM concentrations between groups over time at a significance level of $P=0.05$.

\section{Results}

\section{Study demographics}

In all, eighty three women and their healthy full-term babies completed the study at birth (forty in the $n-3$ fatty acid group and forty-three in the OO group) as reported previously ${ }^{(19)}$. Age, BMI at entry, parity, gestational age, delivery method and socio-economic status were comparable between groups ${ }^{(18)}$. Fig. 1 shows the flow of the participants through the trial. There were no substantial differences between the $n-3$ fatty acid and OO groups in those with blood samples at birth (Table 1). With the exception of birth weight, all other antenatal and birth characteristics were similar in those that provided blood at the 12-year follow-up compared with those that did not participate (online Supplementary Table S1).

\section{Effect of n-3 fatty acid supplementation in pregnant mothers on specialised pro-resolving mediators in childhood}

At birth and at 12 years, measurable concentrations of all SPM except 10 S, 17S-dihydroxydocosahexaenoic acid (DiHDoHE), MaR-1 and PD1 in plasma were detected (Table 2). n-3 Fatty acid supplementation during pregnancy resulted in a 3 -fold increase in cord blood 18-HEPE $(P<0.001)$, a 1.5 -fold increase in 17-HDHA $(P=0.001)$, and increases of $30 \%$ and $20 \%$ for RvE2 $(P=0.008)$ and $\operatorname{RvE} 3(P=0.03)$, respectively, compared with controls. These differences were independent of cord erythrocyte DHA and EPA concentrations. For the groups combined, cord plasma 18-HEPE was significantly positively correlated with cord erythrocyte EPA $(B=151.4, P<0 \cdot 001)$ (Fig. 2(a)). The E-series resolvins (RvE1 and 18R-RvE3), D-series resolvins (RvD1, 17R-RvD1 and RvD2) and 14-HDHA of the

Table 2. Effect of $n-3$ fatty acid supplementation from 20 weeks of gestation till delivery on specialised pro-resolving mediators (SPM) at birth and at 12 years (Geometric means and $95 \%$ confidence intervals)

\begin{tabular}{|c|c|c|c|c|c|c|c|}
\hline \multirow[b]{2}{*}{ SPM (pg/ml) } & \multicolumn{2}{|c|}{$\begin{array}{l}\text { At birth ( } n-3 \text { fatty acids } \\
\quad(n 38) ; \text { OO }(n \text { 43)) }\end{array}$} & \multirow{2}{*}{$\begin{array}{l}\text { Difference between } \\
\text { groups at each time }(P)\end{array}$} & \multicolumn{2}{|c|}{$\begin{array}{l}\text { At } 12 \text { years ( } n-3 \text { fatty acids } \\
\quad(n 24) ; \mathrm{OO}(n 19))\end{array}$} & \multirow{2}{*}{$\begin{array}{l}\text { Difference between } \\
\text { groups at each time }(P)\end{array}$} & \multirow{2}{*}{$\begin{array}{l}\text { Difference in change over time } \\
\left.\text { between groups ( } P_{\text {interaction }}\right)\end{array}$} \\
\hline & Mean & $95 \% \mathrm{Cl}$ & & Mean & $95 \% \mathrm{Cl}$ & & \\
\hline 18-HEPE & & & $<0.001^{*}$ & & & & \\
\hline$n-3$ fatty acids & $301 \cdot 1$ & $261 \cdot 6,346 \cdot 6$ & & $107 \cdot 3$ & $95 \cdot 1,120 \cdot 9$ & 0.232 & $<0.001^{*}$ \\
\hline $\mathrm{OO}$ & 111.2 & $99.6,124.1$ & & $116 \cdot 0$ & $94.8,142 \cdot 0$ & & \\
\hline RvE1 & & & 0.958 & & & & \\
\hline$n-3$ fatty acids & 34.1 & $30.7,37.9$ & & 31.8 & $27 \cdot 7,36 \cdot 5$ & 0.301 & 0.453 \\
\hline OO & 33.4 & $30 \cdot 3,37 \cdot 0$ & & 34.5 & $27 \cdot 4,43 \cdot 4$ & & \\
\hline RvE2 & & & 0.008 & & & & \\
\hline$n-3$ fatty acids & $39.0^{\mathrm{b}}$ & $34.5,44 \cdot 0$ & & $31 \cdot 2^{c}$ & & - & - \\
\hline OO & $30.7^{b}$ & $27 \cdot 7,33 \cdot 9$ & & $29 \cdot 0^{d}$ & & & \\
\hline RvE3 & & & 0.08 & & & & \\
\hline$n-3$ fatty acids & $54 \cdot 4^{\mathrm{e}}$ & $42 \cdot 3,66 \cdot 5$ & & $215 \cdot 5^{\mathrm{g}}$ & & - & - \\
\hline OO & $44 \cdot 2^{f}$ & $29 \cdot 2,59 \cdot 0$ & & $100 \cdot 5^{\mathrm{d}}$ & & & \\
\hline 18R-RvE3 & & & 0.884 & & & & \\
\hline$n-3$ fatty acids & $106 \cdot 8^{\mathrm{h}}$ & $86 \cdot 2,127 \cdot 3$ & & $93 \cdot 8^{\mathrm{j}}$ & $62 \cdot 4,140 \cdot 8$ & 0.102 & 0.119 \\
\hline $\mathrm{OO}$ & $88.0^{i}$ & $70 \cdot 7,109 \cdot 4$ & & 148.4 & $93 \cdot 9,234.6$ & & \\
\hline 17-HDHA & & & $0.001^{*}$ & & & & \\
\hline$n-3$ fatty acids & 699.8 & $607 \cdot 1,806.5$ & & 272.6 & $217 \cdot 7,341 \cdot 4$ & 0.827 & 0.032 \\
\hline OO & $467 \cdot 0$ & $389.5,559.8$ & & 279.9 & $191 \cdot 9$, , $408 \cdot 2$ & & \\
\hline RvD1 & & & 0.259 & & & & \\
\hline$n-3$ fatty acids & $62 \cdot 8$ & $57.7,68.3$ & & $50 \cdot 2$ & $42 \cdot 3,59 \cdot 7$ & 0.618 & 0.938 \\
\hline OO & 67.5 & $61 \cdot 1,74.5$ & & 53.0 & $44 \cdot 3,63.5$ & & \\
\hline 17R-RvD1 & & & 0.166 & & & & \\
\hline$n-3$ fatty acids & 78.0 & $72 \cdot 6,83 \cdot 3$ & & $69 \cdot 8$ & $55 \cdot 8,83.8$ & 0.649 & 0.817 \\
\hline $\mathrm{OO}$ & $73 \cdot 3$ & $67 \cdot 8,78 \cdot 8$ & & $64 \cdot 8$ & $55 \cdot 4,74 \cdot 1$ & & \\
\hline RvD2 & & & 0.264 & & & & \\
\hline$n-3$ fatty acids & $35 \cdot 2$ & $31 \cdot 7,39.0$ & & 28.6 & $25 \cdot 7,31 \cdot 8$ & 0.302 & 0.211 \\
\hline OO & $33 \cdot 4$ & $30 \cdot 2,36 \cdot 8$ & & $30 \cdot 4$ & $27 \cdot 1,34 \cdot 1$ & & \\
\hline 14-HDHA & & & 0.454 & & & 0.969 & \\
\hline$n-3$ fatty acids & $2899 \cdot 0^{\mathrm{h}}$ & $2369.9,3546 \cdot 3$ & & $3462 \cdot 4^{1}$ & $2340 \cdot 4,5122 \cdot 2$ & & 0.709 \\
\hline O० & $2606 \cdot 2^{k}$ & $2114 \cdot 7,3212 \cdot 0$ & & $3499 \cdot 4^{\mathrm{m}}$ & $2208 \cdot 4,5545 \cdot 1$ & & \\
\hline
\end{tabular}

OO, olive oil; HEPE, hydroxyeicosapentaenoic acid; Rv, resolvin; HDHA, hydroxydocosahexaenoic acid.

Data available for: ${ }^{\mathrm{b}} n 35 ;{ }^{\mathrm{c}} n 1 ;{ }^{\mathrm{d}} n 8 ;{ }^{\mathrm{e}} n 36 ;{ }^{\mathrm{f}} n 24 ;{ }^{\mathrm{g}} n 5 ;{ }^{\mathrm{h}} n 37 ;{ }^{\mathrm{i}} n 42 ;{ }^{\mathrm{j}} n 22 ;{ }^{\mathrm{k}} n 41 ;{ }^{\mathrm{I}} n 21 ;{ }^{\mathrm{m}} n 14$

${ }^{*} P$ values remained significant following adjustment for multiple testing. 

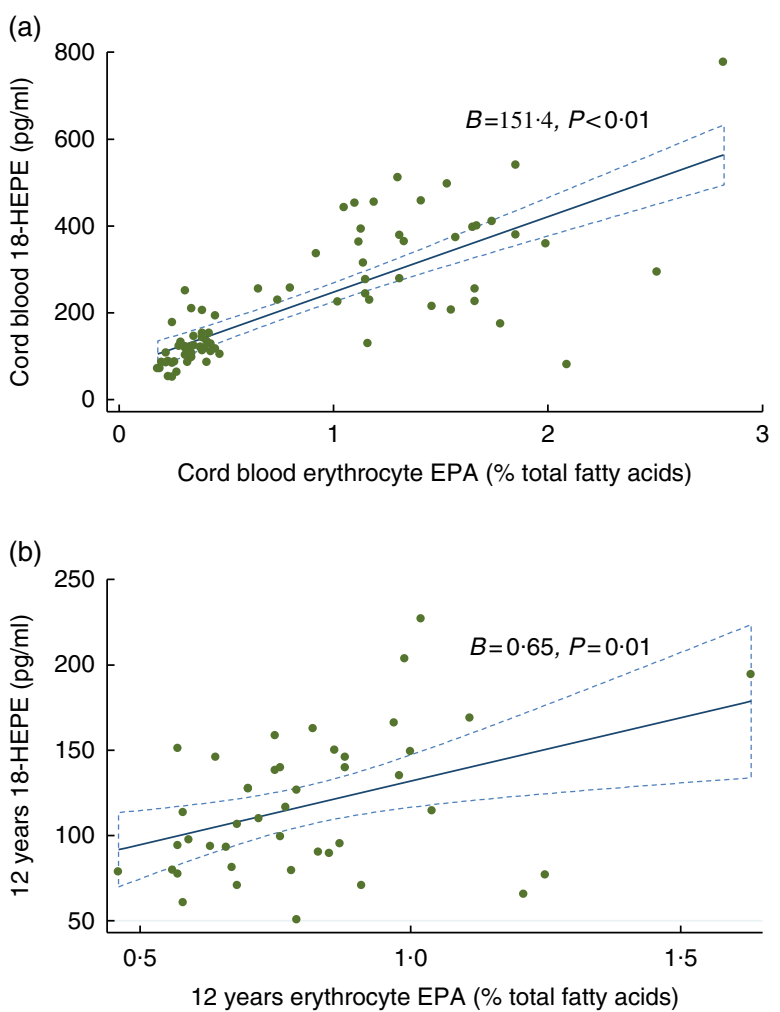

Fig. 2. Associations between 18-hydroxyeicosapentaenoic acid (18-HEPE) and erythrocyte EPA: (a) at birth; (b) at 12 years. ..... , $95 \% \mathrm{Cl}$.

maresin family were not different between the groups at birth (Table 2). The differences between groups for cord blood 18-HEPE and 17-HDHA remained significant following adjustment for multiple comparisons testing.

At 12 years SPM were not different in the offspring of women supplemented with $n-3$ fatty acids compared with the offspring of women who took OO during pregnancy (Table 2). The change in the concentrations of 17-HDHA $\left(P_{\text {interaction }}=0.032\right)($ Fig. 3(a) $)$ and 18-HEPE $\left(P_{\text {interaction }}<0.001\right)$ (Fig. 3(b)) from birth to 12 years was greater in the $n$ - 3 fatty acid group. However, following adjustment for multiple comparisons testing, the change in the concentrations of 17-HDHA over time was no longer significant. For the groups combined, 18-HEPE was significantly positively correlated with erythrocyte EPA at 12 years $(B=0 \cdot 65, P=0 \cdot 01)$ (Fig. 2(b)).

\section{Prevalence of allergic outcomes at 12 months and resolvins at birth}

Table 3 shows the mean SPM concentrations for the allergic and non-allergic participants at 12 months in the $n$ - 3 fatty acid and OO groups, respectively. No formal analysis was performed due to the minimal power to detect interaction effects.

\section{Erythrocyte fatty acid analysis from birth to 12 years}

Erythrocyte EPA, DHA, linoleic acid and arachidonic acid (AA) concentrations were significantly different between the $n-3$ fatty acid and OO groups at birth (in cord blood plasma) (Table 4). However, these differences were not apparent at 12 years (Table 4). The change in the concentrations of EPA $\left(P_{\text {interaction }}<0.001\right)$ and DHA $\left(P_{\text {interaction }}<0.001\right)$ from birth to 12 years was greater in the $n-3$ fatty acid group, which remained significant following adjustment for multiple comparisons testing (Table 4). The change in the concentrations of AA was also significantly different from birth to 12 years before and after adjustments for multiple comparisons testing $\left(P_{\text {interaction }}<0 \cdot 001\right)$

\section{Discussion}

Our study has shown for the first time that supplementing pregnant mothers from 20 weeks' gestation till delivery with $3.7 \mathrm{~g} / \mathrm{d}$ of $n$-3 fatty acids significantly increased several cord blood SPM that have potent biological activity (18-HEPE and 17-HDHA). These differences were not evident in the blood of the offspring at 12 years. We also identified RvE1, RvE2 and RvE3 as well as RvD1, 17R-RvD1 and RvD2 for the first time in human cord blood.

The increase in 18-HEPE and 17-HDHA with $n$-3 fatty acids in our study is important because these SPM are known to promote resolution of inflammation. 18-HEPE and 17-HDHA have been shown to suppress liver tumour formation ${ }^{(34)}$. 18-HEPE has been shown to inhibit neutrophil infiltration in zymosan induced peritonitis ${ }^{(35)}$ and inhibit macrophage-mediated pro-inflammatory activation of cardiac fibroblasts ${ }^{(36)}$. 17-HDHA has also been shown to protect against liver injury ${ }^{(37)}$ and modulate macrophage function to alleviate experimental colitis ${ }^{(38)}$.

We have previously reported in the same study cohort that placental 18-HEPE and 17-HDHA were significantly increased by approximately 3 -fold and 2 -fold, respectively, in the $n$ - 3 fatty acid supplemented group relative to the controls ${ }^{(16)}$. Herein we observed a consistent increase in cord blood 18-HEPE and 17-HDHA with $n$ - 3 fatty acid supplementation and these findings are supported by the fact that both mothers and fetus had significant increase in the proportions of EPA and DHA in the erythrocytes $^{(18)}$. There were consistent coherent relationships between the patterns of SPM production and variations in membrane fatty acid composition. Specifically, cord EPA status was consistently associated with the higher production of 18-HEPE at birth, and this association was also observed between erythrocyte EPA and 18-HEPE at 12 years. This finding is similar to human studies that show that dietary intake of EPA significantly increased plasma concentration of $18-\mathrm{HEPE}^{(36)}$. Our current findings are supported by erythrocyte fatty acid status together with previous reports from the same study that $n-3$ fatty acid supplementation inhibited pro-inflammatory leukotrienes in cord blood-derived neutrophils ${ }^{(39)}$, confirming the antiinflammatory and pro-resolving actions of $n-3$ fatty acids ${ }^{(40)}$.

Our findings are in accordance with previous reports from our group that showed markedly higher concentrations of the resolvin precursors, 18-HEPE and 17-HDHA, in plasma and serum of healthy adults and in patients with chronic kidney disease supplemented with $n-3$ fatty acids ${ }^{(5,33,41)}$. These results also concur with a preliminary report by Mozurkewich 
et al. ${ }^{(42,43)}$ who showed that 17-HDHA significantly increased in maternal and cord blood samples from EPA- or DHA- rich fish oil supplemented participants compared with a soya oil control.
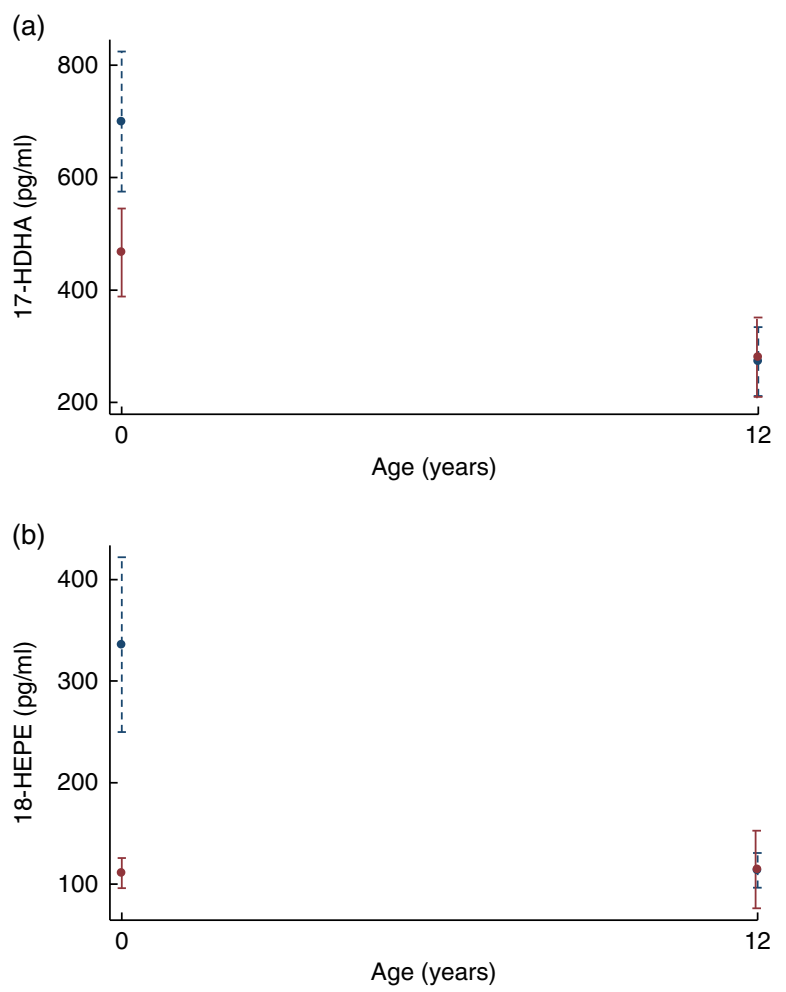

Fig. 3. (a) Plasma resolvin 17-hydroxydocosahexaenoic acid (17-HDHA) concentrations from birth to 12 years. (b) Plasma resolvin 18-hydroxyeicosapentaenoic acid (18-HEPE) concentrations from birth to 12 years. -...- , $n$-3 Fatty acids; ——, OO. Values are predictive margins with $95 \%$ confidence intervals.
In contrast, Mas et al. ${ }^{(5,41)}$ reported an increase in RvD1, RvD2 and 17R-RvD1 in healthy adults following 3 weeks of $n-3$ fatty acid supplementation (35\% EPA and 25\% DHA), whereas Barden et al. ${ }^{(33)}$ showed that supplementing $n-3$ fatty acids $(2.4 \mathrm{~g} / \mathrm{d})$ for $7 \mathrm{~d}$ in healthy adults increased RvE1. Another study supplementing $n-3$ fatty acids $(3.4 \mathrm{~g} / \mathrm{d})$ for 8 weeks in chronic kidney disease patients reported an increase in $\mathrm{RvD}^{(41)}$. It is possible that the steps in further biosynthesis from the intermediates (precursors) to E-series and D-series resolvins may be tightly regulated and may not be subject to increased levels on a relatively short-term (20 weeks) supplementation as used in this study. Our findings highlight that dietary $n-3$ fatty acid supplementation in pregnancy can directly influence the pattern of biologically relevant SPM production in the offspring likely through placental transfer. Increased SPM concentrations in cord blood may be consequent on parturition which is characterised by a pro-inflammatory environment to promote the contraction of the uterus, delivering of the baby and rejection of the placenta ${ }^{(44)}$. Although SPM concentrations were not different between the groups at 12 years, we showed they are still present and may have relevant immunomodulating functions.

While the sample size was insufficient to investigate associations with allergic outcomes in the present data set, future studies may show the importance of SPM in humans in reducing and clearing infections. Pre-clinical models of allergic lung inflammation have shown that RvD1 and RvE1 reduced the consequences of allergic responses by decreasing eosinophil recruitment, type 2 cytokine production and airway hyperresponsiveness, and enhancing allergen phagocytosis and clearance $^{(45-48)}$.

Despite the relatively high concentrations of 14-HDHA of the maresin SPM family in both groups, MaR-1 was not detected in this sample. These findings are in accordance with those of

Table 3. Specialised pro-resolving mediators (SPM) concentrations and the allergic and non-allergic participants by group

(Numbers and percentages; mean values and standard deviations; medians and interquartile ranges (IQR))

\begin{tabular}{|c|c|c|c|c|c|c|c|c|c|c|c|c|c|}
\hline \multirow[b]{3}{*}{ Allergic outcomes at 12 months } & \multicolumn{4}{|c|}{ Prevalence of allergic outcomes } & \multirow[b]{3}{*}{ SPM $(p g / m l)$} & \multicolumn{4}{|c|}{$n-3$ Fatty acids } & \multicolumn{4}{|c|}{$\mathrm{OO}$} \\
\hline & \multicolumn{2}{|c|}{$n$-3 Fatty acids ( $n$ 40) } & \multicolumn{2}{|c|}{$\mathrm{OO}(n 43)$} & & \multicolumn{2}{|c|}{ Allergic } & \multicolumn{2}{|c|}{ Non-allergic } & \multicolumn{2}{|c|}{ Allergic } & \multicolumn{2}{|c|}{ Non-allergic } \\
\hline & $n$ & $\%$ & $n$ & $\%$ & & Mean & SD & Mean & SD & Mean & SD & Mean & SD \\
\hline \multirow[t]{6}{*}{ Atopic eczema dermatitis } & 18 & 45 & 13 & 30 & 18-HEPE & 283.0 & 1.4 & $316 \cdot 6$ & 1.6 & $105 \cdot 0$ & 1.4 & 113.8 & 1.5 \\
\hline & & & & & 17-HDHA & 636.1 & 1.4 & 755.9 & 1.6 & 442.9 & 1.7 & 477.8 & 1.9 \\
\hline & & & & & RvE2 & 39.5 & 1.4 & 38.7 & 1.4 & 28.6 & $1 \cdot 2$ & $31 \cdot 7$ & 1.4 \\
\hline & & & & & RvE3 & & & & & & & & \\
\hline & & & & & Median & \multirow{2}{*}{\multicolumn{2}{|c|}{$\begin{array}{l}48.5 \\
32.6\end{array}$}} & \multirow{2}{*}{\multicolumn{2}{|c|}{$\begin{array}{l}47 \cdot 2 \\
19.8\end{array}$}} & \multirow{2}{*}{\multicolumn{2}{|c|}{$\begin{array}{l}36 \cdot 5 \\
14 \cdot 3\end{array}$}} & \multirow{2}{*}{\multicolumn{2}{|c|}{$\begin{array}{l}29.8 \\
21.4\end{array}$}} \\
\hline & & & & & IQR & & & & & & & & \\
\hline \multirow[t]{6}{*}{ Food allergy } & 3 & 8 & 5 & 12 & 18-HEPE & 236.9 & 1.5 & 307.4 & 1.5 & $89 \cdot 2$ & 1.3 & 114.4 & 1.4 \\
\hline & & & & & 17-HDHA & 796.8 & 1.2 & $692 \cdot 2$ & 1.6 & 386.4 & 1.3 & 478.7 & 1.9 \\
\hline & & & & & RvE2 & 41.8 & 1.7 & 38.7 & 1.4 & 29.7 & 1.4 & $30 \cdot 8$ & 1.3 \\
\hline & & & & & RvE3 & & & & & & & & \\
\hline & & & & & Median & \multirow{2}{*}{\multicolumn{2}{|c|}{$\begin{array}{c}39.9 \\
190.6\end{array}$}} & \multirow{2}{*}{\multicolumn{2}{|c|}{$\begin{array}{l}47 \cdot 8 \\
23 \cdot 4\end{array}$}} & \multirow{2}{*}{\multicolumn{2}{|c|}{$\begin{array}{l}34.9 \\
19.8\end{array}$}} & \multirow{2}{*}{\multicolumn{2}{|c|}{$\begin{array}{l}31 \cdot 7 \\
20 \cdot 8\end{array}$}} \\
\hline & & & & & IQR & & & & & & & & \\
\hline \multirow[t]{6}{*}{ Any allergic disease } & 16 & 40 & 18 & 42 & 18-HEPE & $280 \cdot 2$ & 1.5 & $315 \cdot 6$ & 1.6 & 108.9 & 1.4 & $112 \cdot 8$ & 1.4 \\
\hline & & & & & 17-HDHA & 640.4 & 1.4 & 741.4 & 1.6 & 447.9 & 1.7 & $481 \cdot 1$ & 1.9 \\
\hline & & & & & RvE2 & $40 \cdot 7$ & 1.4 & 38.0 & 1.4 & 29.4 & $1 \cdot 2$ & 31.4 & 1.4 \\
\hline & & & & & RvE3 & & & & & & & & \\
\hline & & & & & Median & 48. & & 47 & & 13 & & 30 & \\
\hline & & & & & IQR & 32. & & 19 & & 14. & & 21 & \\
\hline
\end{tabular}

OO, olive oil; HEPE, hydroxyeicosapentaenoic acid; HDHA, hydroxydocosahexaenoic acid; Rv, resolvin. 
Table 4. Comparison of groups for erythrocyte fatty acid concentrations at birth and 12 years (Mean values, geometric means and standard deviations)

\begin{tabular}{|c|c|c|c|c|c|c|c|}
\hline \multirow{2}{*}{$\begin{array}{l}\text { Fatty acids (\% total } \\
\text { fatty acids) }\end{array}$} & \multicolumn{2}{|l|}{ At birth } & \multirow{2}{*}{$\begin{array}{c}\text { Difference between groups at } \\
\text { each time }(P) \dagger\end{array}$} & \multicolumn{2}{|c|}{ At 12 years } & \multirow{2}{*}{$\begin{array}{c}\text { Difference between groups } \\
\text { at each time }(P) \dagger\end{array}$} & \multirow{2}{*}{$\begin{array}{c}\text { Difference in change over time between } \\
\text { groups }\left(P_{\text {interaction })} \dagger\right.\end{array}$} \\
\hline & Mean & SD & & Mean & $\mathrm{SD}$ & & \\
\hline \multicolumn{8}{|l|}{ EPA $(20: 5 n-3)$} \\
\hline$n-3$ fatty acids & \multirow{2}{*}{\multicolumn{2}{|c|}{$1.21^{\mathrm{d}}$}} & \multirow{3}{*}{$<0.001^{*}$} & \multirow{2}{*}{\multicolumn{2}{|c|}{$0.74^{f}$}} & 0.081 & $<0.001^{*}$ \\
\hline Geometric mean & & & & & & & \\
\hline SD & \multicolumn{2}{|l|}{1.6} & & \multicolumn{2}{|c|}{1.2} & & \\
\hline \multicolumn{8}{|l|}{ OO } \\
\hline Geometric mean & \multirow{2}{*}{\multicolumn{2}{|c|}{$0.33^{\mathrm{e}}$}} & \multicolumn{3}{|c|}{$0.84^{\mathrm{g}}$} & & \\
\hline SD & & & \multicolumn{3}{|c|}{1.3} & & \\
\hline \multicolumn{8}{|l|}{ DHA $(22: 6 n-3)$} \\
\hline$n-3$ fatty acids & $10 \cdot 2^{\mathrm{d}}$ & $1 \cdot 1$ & \multirow[t]{2}{*}{$<0.001^{*}$} & $4 \cdot 65^{f}$ & 0.7 & \multirow[t]{2}{*}{0.26} & \multirow[t]{2}{*}{$<0.001^{*}$} \\
\hline OO & $7 \cdot 3^{\mathrm{e}}$ & 0.9 & & $4 \cdot 34^{\mathrm{g}}$ & 1.0 & & \\
\hline \multicolumn{8}{|l|}{ Linoleic acid $(18: 2 n-6)$} \\
\hline$n-3$ fatty acids & $3 \cdot 84^{d}$ & 0.5 & \multirow[t]{2}{*}{$0.007^{\star}$} & $11 \cdot 3^{f}$ & 1.0 & \multirow[t]{2}{*}{0.14} & \multirow[t]{2}{*}{0.59} \\
\hline $\mathrm{OO}$ & $3.59^{e}$ & 0.4 & & $11 \cdot 0^{\mathrm{g}}$ & 0.8 & & \\
\hline \multicolumn{8}{|c|}{ Arachidonic acid $(20: 4 n-6)$} \\
\hline$n-3$ fatty acids & $15 \cdot 0^{d}$ & 1.4 & \multirow[t]{2}{*}{$<0.001^{*}$} & $16 \cdot 3^{f}$ & 0.9 & \multirow[t]{2}{*}{0.05} & \multirow[t]{2}{*}{$<0.001^{*}$} \\
\hline $\mathrm{OO}$ & $17 \cdot 4^{\mathrm{e}}$ & 1.2 & & $16.9^{\mathrm{g}}$ & 0.8 & & \\
\hline
\end{tabular}

Data available for: ${ }^{\mathrm{d}} n 39,{ }^{\mathrm{e}} n 43,{ }^{\mathrm{f}} n 24,{ }^{\mathrm{g}} n 19$.

* $P$ values remained significant following adjustment for multiple testing.

$\dagger P$ values obtained using linear mixed models with maximum likelihood estimation.

Barden et $a l .{ }^{(33)}$ in healthy adults supplemented with $n$-3 fatty acids. We did not detect 10S,17S-DiHDoHE or MaR-1 in cord blood following $n-3$ fatty acid supplementation, possibly suggesting a preferential synthesis of D-series resolvins from DHA during pregnancy. The non-detection of PD1 could be due to this SPM being converted by leucocytes to an $n$-22 monohydroxylated metabolite of PD1, which retains biological function with macrophages ${ }^{(49,50)}$.

A major strength of our study is the comprehensive antenatal and offspring data on the participants and their follow-up to 12 years of age. Samples for analysis of SPM at 12 years were recently collected whereas cord plasma samples had been stored long-term at $-80^{\circ} \mathrm{C}$ before analysis, which could be a limitation of this study. Although statistical analyses employed MLE that accounts for missing data, we acknowledge the high attrition rate at 12 years could be a further limitation. The length of the dietary intervention may be another important factor as the impact of $n-3$ fatty acid consumption may require a longer and ongoing intervention period in order to detect a difference in SPM at 12 years of age.

Despite this, this is the first report of 18-HEPE in cord blood plasma following maternal supplementation with $n-3$ fatty acids. Our results show that $n-3$ fatty acids in pregnancy do in fact raise cord SPM concentrations. Although the study was not powered to detect any associations with allergic outcomes, SPM at birth may be relevant to the alleviation of low-grade inflammatory status associated with pregnancy which may be beneficial to the fetus. These findings add significantly to the very limited literature reporting SPM in humans, particularly in children. However, $n-3$ fatty acid supplementation in pregnancy on SPM are not long-lasting beyond the end of supplementation. Further studies are required to explore the biological effects of SPM in cord blood to ascertain their significance in mediating the beneficial effects of $n-3$ fatty acid supplementation at birth and their pharmacological applications in relation to longer-term modification of health and disease in children.

\section{Acknowledgements}

The authors thank the staff and participants who helped in this study. The authors particularly thank Carlie Dunford for her assistance with recruitment.

This work was supported by the National Health and Medical Research Council of Australia (NH\&MRC) (grant no. APP1010495). The NH\&MRC had no role in the design, analysis or writing of this article.

T. A. M., S. L. P., L. J. B. and R.-C. H.: designed and supervised the study; V. H. L. S.: conducted the study and had primary responsibility for the final content; V. H. L. S. and S. B.: analysed the data; V. H. L. S., E. M., S. L. P., L. J. B., A. E. B., T. A. M. and R.-C. H.: wrote the manuscript. The study sponsors were not involved in the design, implementation, analysis, presentation or interpretation of results.

\section{References}

1. Serhan CN \& Petasis NA (2011) Resolvins and protectins in inflammation resolution. Chem Rev 111, 5922-5943.

2. Bannenberg G \& Serhan CN (2010) Specialized pro-resolving lipid mediators in the inflammatory response: an update. Biochim Biophys Acta 12, 10.

3. Tabas I (2010) Macrophage death and defective inflammation resolution in atherosclerosis. Nat Rev Immunol 10, 36-46.

4. Serhan CN, Chiang N, Dalli J, et al. (2014) Lipid mediators in the resolution of inflammation. Cold Spring Harb Perspect Biol 7, a016311.

5. Mas E, Croft KD, Zahra P, et al. (2012) Resolvins D1, D2, and other mediators of self-limited resolution of inflammation in human blood following $n-3$ fatty acid supplementation. Clin Chem 58, 1476-1484.

6. Morita M, Kuba K, Ichikawa A, et al. (2013) The lipid mediator Protectin D1 inhibits influenza virus replication and improves severe influenza. Cell 153, 112-125. 
7. Lima-Garcia JF, Dutra RC, da Silva K, et al. (2011) The precursor of resolvin $\mathrm{D}$ series and aspirin-triggered resolvin D1 display anti-hyperalgesic properties in adjuvant-induced arthritis in rats. Br J Pharmacol 164, 278-293.

8. Huang L, Wang C-F, Serhan CN, et al. (2011) Enduring prevention and transient reduction of postoperative pain by intrathecal resolvin D1. Pain 152, 557-565.

9. Bento AF, Claudino RF, Dutra RC, et al. (2011) Omega-3 fatty acid-derived mediators 17(R)-hydroxy docosahexaenoic acid, aspirin-triggered resolvin D1 and resolvin D2 prevent experimental colitis in mice. J Immunol 187, 1957-1969.

10. Koltsida O, Karamnov S, Pyrillou K, et al. (2013) Toll-like receptor 7 stimulates production of specialized pro-resolving lipid mediators and promotes resolution of airway inflammation. EMBO Mol Med 5, 762-775.

11. Krishnamoorthy N, Burkett PR, Dalli J, et al. (2015) Cutting edge: Maresin-1 engages regulatory $\mathrm{T}$ cells to limit type 2 innate lymphoid cell activation and promote resolution of lung inflammation. J Immunol 194, 863-867.

12. de Paiva CS, Schwartz CE, Gjorstrup P, et al. (2012) Resolvin E1 (RX-10001) reduces corneal epithelial barrier disruption and protects against goblet cell loss in a murine model of dry eye. Cornea 31, 1299-1303.

13. Dalli J, Kraft BD, Colas RA, et al. (2015) Proresolving lipid mediator profiles in baboon pneumonia are regulated by inhaled carbon monoxide. Am J Resp Cell Mol 53, 314-325.

14. Dalli J, Ramon S, Norris PC, et al. (2015) Novel proresolving and tissue-regenerative resolvin and protectin sulfidoconjugated pathways. FASEB J 29, 2120-2136

15. Jones ML, Mark PJ, Keelan JA, et al. (2013) Maternal dietary omega-3 fatty acid intake increases resolvin and protectin levels in the rat placenta. J Lipid Res 54, 2247-2254.

16. Keelan JA, Mas E, D'Vaz N, et al. (2015) Effects of maternal $n-3$ fatty acid supplementation on placental cytokines, pro-resolving lipid mediators and their precursors. Reproduction 149, 171-178.

17. Barker DJP (2004) The developmental origins of adult disease. J Am Coll Nutr 23, Suppl. 6, 588S-595S.

18. Dunstan JA, Mori TA, Barden A, et al. (2004) Effects of $n-3$ polyunsaturated fatty acid supplementation in pregnancy on maternal and fetal erythrocyte fatty acid composition. Eur J Clin Nutr 58, 429-437.

19. Dunstan JA, Mori TA, Barden A, et al. (2003) Fish oil supplementation in pregnancy modifies neonatal allergenspecific immune responses and clinical outcomes in infants at high risk of atopy: a randomized, controlled trial. J Allergy Clin Immunol 112, 1178-1184.

20. Dunstan JA, Mori TA, Barden A, et al. (2003) Maternal fish oil supplementation in pregnancy reduces interleukin-13 levels in cord blood of infants at high risk of atopy. Clin Exp Allergy 33, 442-448.

21. Denburg JA, Hatfield HM, Cyr MM, et al. (2005) Fish oil supplementation in pregnancy modifies neonatal progenitors at birth in infants at risk of atopy. Pediatr Res 57, 276-281.

22. Barden AE, Mori TA, Dunstan JA, et al. (2004) Fish oil supplementation in pregnancy lowers $\mathrm{F}_{2}$-isoprostanes in neonates at high risk of atopy. Free Radic Res 38, 233-239.

23. Dunstan JA, Simmer K, Dixon G, et al. (2008) Cognitive assessment of children at age $2^{1 / 2}$ years after maternal fish oil supplementation in pregnancy: a randomised controlled trial. Arch Dis Child Fetal Neonatal Ed 93, F45-F50.

24. Dunstan JA, Roper J, Mitoulas L, et al. (2004) The effect of supplementation with fish oil during pregnancy on breast milk immunoglobulin A, soluble CD14, cytokine levels and fatty acid composition. Clin Exp Allergy 34, 1237-1242.
25. Dunstan JA, Mitoulas LR, Dixon G, et al. (2007) The effects of fish oil supplementation in pregnancy on breast milk fatty acid composition over the course of lactation: a randomized controlled trial. Pediatr Res 62, 689-694.

26. Olsen SF, Dalby S $\phi$ rensen J, Secher NJ, et al. (1992) Randomised controlled trial of effect of fish-oil supplementation on pregnancy duration. Lancet 339, 1003-1007.

27. Onwude JL, Lilford RJ, Hjartardottir H, et al. (1995) A randomised double blind placebo controlled trial of fish oil in high risk pregnancy. Br J Obstet Gynaecol 102, 95-100.

28. Salvig JD, Olsen SF \& Secher NJ (1996) Effects of fish oil supplementation in late pregnancy on blood pressure: a randomised controlled trial. Br J Obstet Gynaecol 103, 529-533.

29. Mori TA, Beilin LJ, Burke V, et al. (1997) Interactions between dietary fat, fish, and fish oils and their effects on platelet function in men at risk of cardiovascular disease. Arterioscler Thromb Vasc Biol 17, 279-286.

30. Mori TA, Burke V, Puddey IB, et al. (2000) Purified eicosapentaenoic and docosahexaenoic acids have differential effects on serum lipids and lipoproteins, LDL particle size, glucose, and insulin in mildly hyperlipidemic men. Am J Clin Nutr 71, 1085-1094.

31. Pottala J, Espeland M, Polreis J, et al. (2012) Correcting the effects of $-20{ }^{\circ} \mathrm{C}$ storage and aliquot size on erythrocyte fatty acid content in the Women's Health Initiative. Lipids $\mathbf{4 7}$, 835-846.

32. Mas E, Croft KD, Zahra P, et al. (2012) Resolvins D1, D2, and other mediators of self-limited resolution of inflammation in human blood following $n-3$ fatty acid supplementation. Clin Chem 58, 1476-1484.

33. Barden A, Mas E, Croft KD, et al. (2014) Short-term $n$-3 fatty acid supplementation but not aspirin increases plasma proresolving mediators of inflammation. J Lipid Res 55, 2401-2407.

34. Weylandt KH, Krause LF, Gomolka B, et al. (2011) Suppressed liver tumorigenesis in fat-1 mice with elevated omega-3 fatty acids is associated with increased omega- 3 derived lipid mediators and reduced TNF-alpha. Carcinogenesis 32, 897-903.

35. Isobe $\mathrm{Y}$, Arita M, Matsueda S, et al. (2012) Identification and structure determination of novel anti-inflammatory mediator resolvin E3, 17,18-dihydroxyeicosapentaenoic acid. J Biol Chem 287, 10525-10534.

36. Endo J, Sano M, Isobe Y, et al. (2014) 18-HEPE, an n-3 fatty acid metabolite released by macrophages, prevents pressure overload-induced maladaptive cardiac remodeling. J Exp Med 211, 1673-1687.

37. González-Périz A, Planagumà A, Gronert K, et al. (2006) Docosahexaenoic acid (DHA) blunts liver injury by conversion to protective lipid mediators: protectin D1 and 17S-hydroxy-DHA. FASEB J 20, 2537-2539.

38. Chiu CY, Gomolka B, Dierkes C, et al. (2012) Omega-6 docosapentaenoic acid-derived resolvins and 17-hydroxydocosahexaenoic acid modulate macrophage function and alleviate experimental colitis. Inflamm Res 61, 967-976.

39. Prescott SL, Barden AE, Mori TA, et al. (2007) Maternal fish oil supplementation in pregnancy modifies neonatal leukotriene production by cord-blood-derived neutrophils. Clin Sci 113, 409-416.

40. Mori T \& Beilin L (2004) Omega-3 fatty acids and inflammation. Curr Atheroscler Rep 6, 461-467.

41. Mas E, Barden A, Burke V, et al. (2015) A randomized controlled trial of the effects of $n$ - 3 fatty acids on resolvins in chronic kidney disease. Clin Nutr 35, 331-336.

42. Mozurkewich EL, Clinton CM, Chilimigras JL, et al. (2013) The mothers, omega-3, and mental health study: a double-blind, randomized controlled trial. Am J Obstet Gynecol 208, 313. e311-313.e319. 
43. Mozurkewich E, Greenwood M, Romero V, et al. (2015) 792: EPA- and DHA-rich fish oil supplementation augments maternal and cord blood resolvin pathway markers: a mothers, omega-3, \& mental health study secondary analysis. Am J Obstet Gynecol 212, S383-S384.

44. Mor G, Cardenas I, Abrahams V, et al. (2011) Inflammation and pregnancy: the role of the immune system at the implantation site. Ann N Y Acad Sci 1221, 80-87.

45. Levy BD, Kohli P, Gotlinger K, et al. (2007) Protectin D1 is generated in asthma and dampens airway inflammation and hyperresponsiveness. J Immunol 178, 496-502.

46. Haworth O, Cernadas M, Yang R, et al. (2008) Resolvin E1 regulates interleukin 23, interferon-gamma and lipoxin A4 to promote the resolution of allergic airway inflammation. Nat Immunol 9, 873-879.
47. Aoki H, Hisada T, Ishizuka T, et al. (2008) Resolvin E1 dampens airway inflammation and hyperresponsiveness in a murine model of asthma. Biochem Biophys Res Commun 367, 509-515.

48. Rogerio AP, Haworth O, Croze R, et al. (2012) Resolvin D1 and aspirin-triggered resolvin D1 promote resolution allergic airways responses. J Immunol 189, 1983-1991.

49. Connor KM, SanGiovanni JP, Lofqvist C, et al. (2007) Increased dietary intake of omega-3-polyunsaturated fatty acids reduces pathological retinal angiogenesis. Nat Med 13, 868-873.

50. Tungen JE, Aursnes M, Vik A, et al. (2014) Synthesis and anti-inflammatory and pro-resolving activities of 22-OH-PD1, a monohydroxylated metabolite of protectin D1. J Nat Prod 77, 2241-2247. 